\title{
Minimizing Drilling Thrust Force for HFRP Composite by Optimizing Process Parameters using Combination of ANOVA Approach and S/N Ratios Analysis
}

\author{
Mohd Azuwan Maoinser ${ }^{1, a}$, Faiz Ahmad ${ }^{1, b^{*}}$, Safian Sharif ${ }^{2, c^{*}}$ and Woo Tze Keong ${ }^{1, d^{*}}$ \\ ${ }^{1}$ Mechanical Engineering Department, Universiti Teknologi PETRONAS, Bandar Seri Iskandar, \\ 31750, Tronoh, Perak \\ ${ }^{2}$ Manufacturing and Industrial Engineering Department, Faculty of Mechanical Engineering, \\ Universiti Teknologi Malaysia, 81310, Skudai, Johor, Malaysia
}

\begin{abstract}
Drilling hybrid fiber reinforced polymer (HFRP) composite is a novel approach in fiber reinforced polymer (FRP) composite machining studies as this material combining two different fibers in a single matrix that resulted in considerable improvement in mechanical properties and cost saving as compared to conventional fiber composite material. This study presents the development and optimized way of drilling HFRP composite at various drilling parameters such as drill point angle, feed rate and cutting speed by using the full factorial design experiment with the combination of analysis of variance (ANOVA) approach and signal to noise $(\mathrm{S} / \mathrm{N})$ ratio analysis. The results identified optimum drilling parameters for drilling the HFRP composite using small drill point angle at low feed rate and medium cutting speed that resulted in lower thrust force.
\end{abstract}

\section{Introduction}

Hybrid fiber reinforced polymer (HFRP) composite is an advanced fiber reinforced polymer (FRP) composite that used two or more different fiber reinforcement in a polymer matrix. Advantage of using this composite is that it is possible to improve the failure strain by incorporated high elongation fiber like glass fiber into low elongation fiber such as carbon fiber [1]. This significant advantage had encouraged the application of HFRP composite on various structures such as bridge, drain grating covers and oil and gas platform. Holes are always required in these applications either for mechanical fastening purpose or other structural requirement like fluid drainage function. However, drilling holes on the fiber reinforced polymer (FRP) composite using conventional drilling draw a great attention as it will damage the material's structure due to the unsuitable drilling method such as the selection of incorrect drill geometry and improper selection of drilling parameters.

aazuwan_173@yahoo.com, ${ }^{\mathrm{b}}$ faizahmad@petronas.com.my, ${ }^{\mathrm{c}}$ safian@fkm.utm.my, ${ }^{\mathrm{d}} \mathrm{kenz}$ (8@gmail.com 
Abrão et al. [2] have studied the effect of the cutting tool geometry and material on the thrust force and delamination produced when drilling a glass fiber reinforced epoxy composite. They concluded that "Brad and Spur" carbide drill with two cutting edges provide the lowest thrust force that is responsible for the least damage on the composite laminate. Durão et al. [3] did a comparative study on different drill point geometry and feed rate for drilling carbon fiber reinforced laminates. The used of $120^{\circ}$ drill point geometry had produced the minimum delamination factor $\left(F_{\mathrm{d}}\right)$ when drilling the composite at higher feed rate. Heisel et al. [4] investigated the influence of a drill tool at increased cutting speed on machining forces and drill hole quality (delamination, fraying and burr formation) of carbon fiber reinforced polymer (CFRP) composite. Their assessment shows that the quality of holes at entrance is best when using point angle $>180^{\circ}$, while it is poor at the exit.

In FRP composite drilling study, thrust force may or may not influence the delamination occur at the drilled holes area. Khashaba [5] have studied the relationship between the thrust force and delamination in drilling glass fiber reinforced (GFR)-thermoset composites. He concluded that the delamination increased with increasing thrust force. On the other hand, Abrão et al. [2] found out that the relationship between thrust force and delamination is not a straightforward as he studied the effect of the cutting tool geometry on thrust force and delamination when drilling glass fiber reinforced plastic composites.

The research conduct on [2-5] were mostly related to drilling the conventional FRP composite materials like glass fiber reinforced polymer (GFRP) composite or carbon fiber reinforced polymer (CFRP) composite using various drill point angle, cutting speed and feed rate. None of them have studied on the drilling of hybrid fiber reinforced polymer (HFRP) composite. Therefore, the objective of this study is to optimize the drilling parameters for drilling holes in HFRP composite through thrust force minimization using full factorial design experiment with the aid of analysis of variance (ANOVA) and signal to noise $(\mathrm{S} / \mathrm{N})$ ratio analysis.

\section{Experimental Methodologies}

\subsection{Fabrication of HFRP Composite}

In this study, hybrid fiber reinforced polymer (HFRP) composite was fabricated using the vacuum infusion molding (VIM) technique. Vacuum infusion or VI (a.k.a. VARTM, VM, RIFT and SCRIMP ${ }^{\mathrm{TM}}$ ) has the ability to reduce costs by increasing predictability and ultimately achieving consistent production [6]. The consistent production of HFRP composite is very important in this study to ensure the damage observed after drilling work is not influenced by the manufacturing flaws. Fig. 1 shows the setup of VIM technique used to fabricate the HFRP composite.

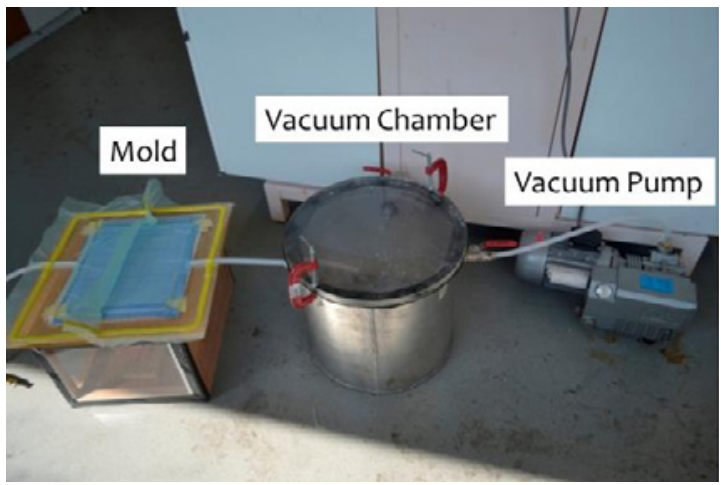

Figure 1. Vacuum infusion molding (VIM) setup used to fabricate HFRP composite 
The used of vacuum infusion molding (VIM) technique together with the stacking sequence of $\left[(0 / 90)_{\mathrm{c}}(0 / 90)_{\mathrm{g}}(0 / 90)_{\mathrm{g}}(0 / 90)_{\mathrm{g}}(0 / 90)_{\mathrm{c}}\right]_{4 \mathrm{~s}}$ resulted in $45 \%$ fiber volume fraction (FVF) of HFRP composite. Epoxy resin (infusion grade) is used as the polymer matrix to incorporate the glass fiber and carbon fiber that was pressurized in $-95 \mathrm{kPa}$ vacuum pressures and post cure in the oven at $90^{\circ} \mathrm{C}$ for two hours. This laminates was tested according to ASTM standard method for its physical and mechanical properties as shown in Table 1.

Table 1 Physical and mechanical properties of HFRP composite laminates

\begin{tabular}{|l|l|c|}
\hline ASTM Standard & \multicolumn{2}{|c|}{ Properties Test } \\
\hline ASTM D 0792-00 & Density [g/cm $\left.{ }^{3}\right]$ & 1.66 \\
\hline ASTM D 3173-00 & Fiber Volume Fraction [\%] & 45 \\
\hline ASTM D 3039-00 & Tensile Strength [MPa] & 419 \\
\hline ASTM D 2344 -00 & ILSS [MPa] & 62 \\
\hline ASTM D 0790-03 & Flexural Strength [MPa] & 246 \\
\hline
\end{tabular}

\subsection{Drilling of HFRP Composite}

The study on drilling HFRP composite laminates is considered as a novel approach in composites drilling area when no significant study was found in the literature research. Thus, various drill point angle, cutting speed and feed rate which considered as a fundamental way in drilling process are used to accomplish the objective of this study. Computer numerical control (CNC) Mazak Variaxis 630-5X with $30 \mathrm{~kW}$ power and a maximum spindle speed of $25000 \mathrm{rpm}$ is used to drill the HFRP composite at various drilling parameters. Table 2 shows the drilling parameters used to drill the HFRP composite in this study.

Table 2 Drilling parameters used to drill the HFRP composite in this study

\begin{tabular}{|c|c|c|c|}
\hline Level & Drill Point Angle $\left[{ }^{\circ}\right]$ & Cutting Speed $[\mathrm{m} / \mathrm{min}]$ & Feed Rate $[\mathrm{mm} / \mathrm{rev}]$ \\
\hline 1 & 85 & 53 & 0.02 \\
\hline 2 & 118 & 106 & 0.06 \\
\hline 3 & 135 & 212 & 0.12 \\
\hline
\end{tabular}

Recently, solid carbide drill is used as a substitute polycrystalline diamond (PCD) drill and high speed steel (HSS) drill in FRP composite drilling study. It is due to the ability of this tool to overcome the immediate deformation resulted from increasing cutting force and cutting temperature during drilling [7]. In this study, K200 solid carbide twist drill is used to drill the HFRP composite. As the drill point of this drill is varied to three different angles, the other drill geometry such as helix angle, chisel edge angle, web thickness and diameter is kept constant as shown in Table 3.

Table 3 Specifications of drill bit used to drill the HFRP composite in this study

\begin{tabular}{|l|c|}
\hline Drill point angle $\left({ }^{\circ}\right)$ & $85,118,135$ \\
\hline Material & K 200 Solid Carbide \\
\hline Diameter $(\mathrm{mm})$ & 6 \\
\hline Helix Angle $\left(^{\circ}\right)$ & 25 \\
\hline Chisel edge angle $\left(^{\circ}\right)$ & 143 \\
\hline Web thickness $(\mathrm{mm})$ & 0.63 \\
\hline
\end{tabular}




\subsection{Measurement on Thrust Force of Drilling HFRP composite}

Fig. 2 shows how the Piezoelectric Dynamometer 9257B was set up to measure the thrust force in drilling HFRP composite. It was connected to multichannel amplifier 9017B and computer for data acquisition and processing. In fact, this dynamometer was purposely designed for measuring the turning force. However, it has been modified so that the thrust force of drilling HFRP composite laminates can be measured. The modification of this dynamometer included the replacement of the tool holder for turning force measurement with the attachment of two aluminum plate where HFRP composite sample was put between it. These aluminum plates was also act as back-up plate where five holes with $10 \mathrm{~mm}$ diameter so that the drill allows for larger critical thrust force where larger feed rate can be operated without delamination damage [8]. Fig. 3 shows the modification of the dynamometer 9257B.

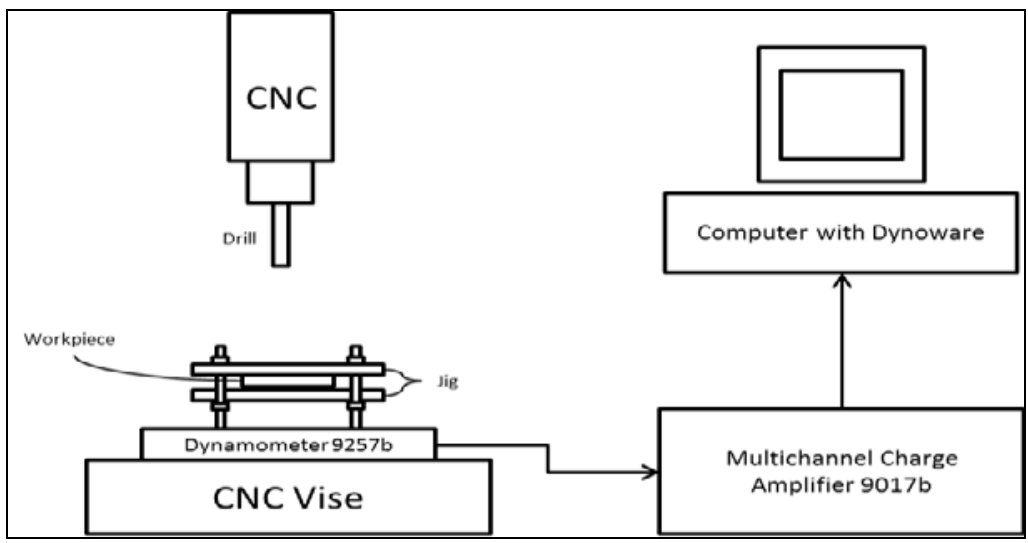

Figure 2 Thrust force evaluation setup in drilling HFRP composite
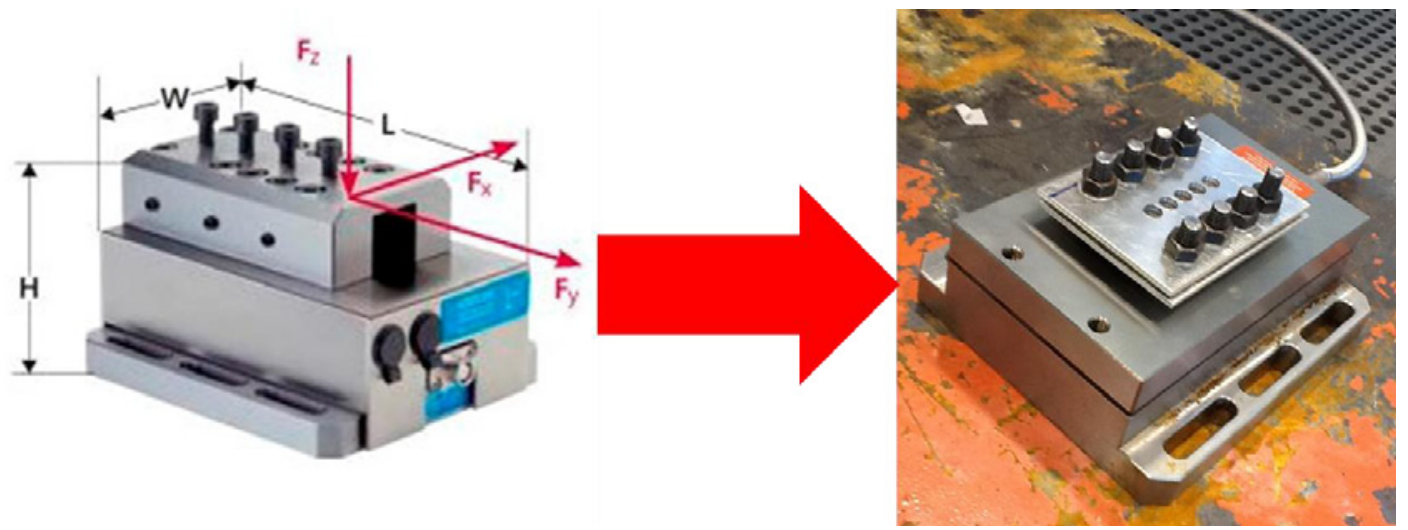

Figure 3 Modification of Dynamometer 9257B

\subsection{Design of Experiments}

In this study, full factorial design experiments is used to find the optimization of drilling HFRP composites based on thrust force and delamination factor analysis. As mentioned earlier in Table 2, three levels of each drilling parameters (drill point angle, cutting speed and feed rate) are used in this 
study. Each level is replicated five times where average value of these five readings is taken for the accuracy of the data.

The experimental result of this study is transformed into the signal to noise $(\mathrm{S} / \mathrm{N})$ ratio analysis. This combination between full factorial design experiment and $\mathrm{S} / \mathrm{N}$ ratio analysis was employed from Mohan et al. [9]. The used of the $\mathrm{S} / \mathrm{N}$ ratio analysis recommended by Taguchi is to measure the quality characteristics that deviated from the desired results where greater $\mathrm{S} / \mathrm{N}$ ratio corresponds to the better quality characteristics regardless of the category of quality characteristics [10]. There are three categories of the quality characteristics in the analysis of $\mathrm{S} / \mathrm{N}$ ratio, i.e. the-lower-the-better, thehigher-the-better, and the-nominal-the-better. The following equations (Eq. 2, Eq. 3 and Eq. 4) show the three categories of $\mathrm{S} / \mathrm{N}$ ratio analysis.

Nominal is the best characteristic:

$$
\frac{S}{N}=10 \log \frac{\overline{\mathrm{y}}}{s_{y}^{2}}
$$

Smaller is the better characteristic:

$$
\frac{S}{N}=-10 \log \frac{1}{n}\left(\Sigma y^{2}\right)
$$

Larger the better characteristic:

$$
\frac{S}{N}=-\log \frac{1}{n}\left(\Sigma \frac{1}{y^{2}}\right)
$$

where $\bar{y}$ is the average of the observed data, $s_{y}^{2}$ the variation of $\mathrm{y}, n$ the number observations, and $y$ is the observed data. Since the response (thrust force) in this study should be minimized in order to achieve the optimization of the drilling parameters, smaller is the better characteristics (Eq. 3) is chosen to meet this objective [11].

\section{Results and Discussion}

\subsection{Effect of drill point angle on thrust force}

In this study, analysis of variance (ANOVA) is used to analyze which factors has influenced on the thrust force when drilling HFRP composite at various drilling parameters (drill point angle, cutting speed and feed rate). Table 4 and 5 indicate that feed rate and drill point angle has the significant influence on the drilling thrust following by the cutting speed. In fact, this significant influence are proven in Fig. 5 as it shows a large gradient slope of the feed rate and drill point angle in main effect plots for $\mathrm{S} / \mathrm{N}$ ratios. Furthermore, this figure shows that it is possible to achieve minimum thrust force by drilling the HFRP composite at low feed rate $(0.02 \mathrm{~mm} / \mathrm{rev})$, small drill point angle $\left(85^{\circ}\right)$ and medium cutting speed $(106 \mathrm{~m} / \mathrm{min})$.

Table 4 Analysis of variance (ANOVA) for SN ratios (thrust force)

\begin{tabular}{|l|c|c|c|c|c|c|}
\hline Source & DF & Seq SS & Adj SS & Adj MS & F & P \\
\hline Point Angle $\left(^{\circ}\right)$ & 2 & 185.65 & 185.65 & 92.8238 & 103.95 & 0.000 \\
\hline Cutting Speed (m/min) $^{\circ}$ & 2 & 13.46 & 13.46 & 6.7277 & 7.53 & 0.004 \\
\hline Feed Rate (mm/rev) & 2 & 177.78 & 177.78 & 88.8907 & 99.55 & 0.000 \\
\hline Residual Error & 20 & 17.86 & 17.86 & 0.8929 & & \\
\hline Total & 26 & 394.74 & & & & \\
\hline
\end{tabular}


Table 5 Response table for signal to noise $(\mathrm{S} / \mathrm{N})$ ratios smaller is better (thrust force)

\begin{tabular}{|c|c|c|c|}
\hline Level & Point Angle $\left(^{\circ}\right)$ & Cutting Speed (m/min) & Feed Rate (mm/rev) \\
\hline $\mathbf{1}$ & -19.23 & -23.88 & -19.45 \\
\hline $\mathbf{2}$ & -24.19 & -22.26 & -23.61 \\
\hline $\mathbf{3}$ & -25.25 & -22.53 & -25.61 \\
\hline Delta & 6.01 & 1.61 & 6.16 \\
\hline Rank & 2 & 3 & 1 \\
\hline
\end{tabular}

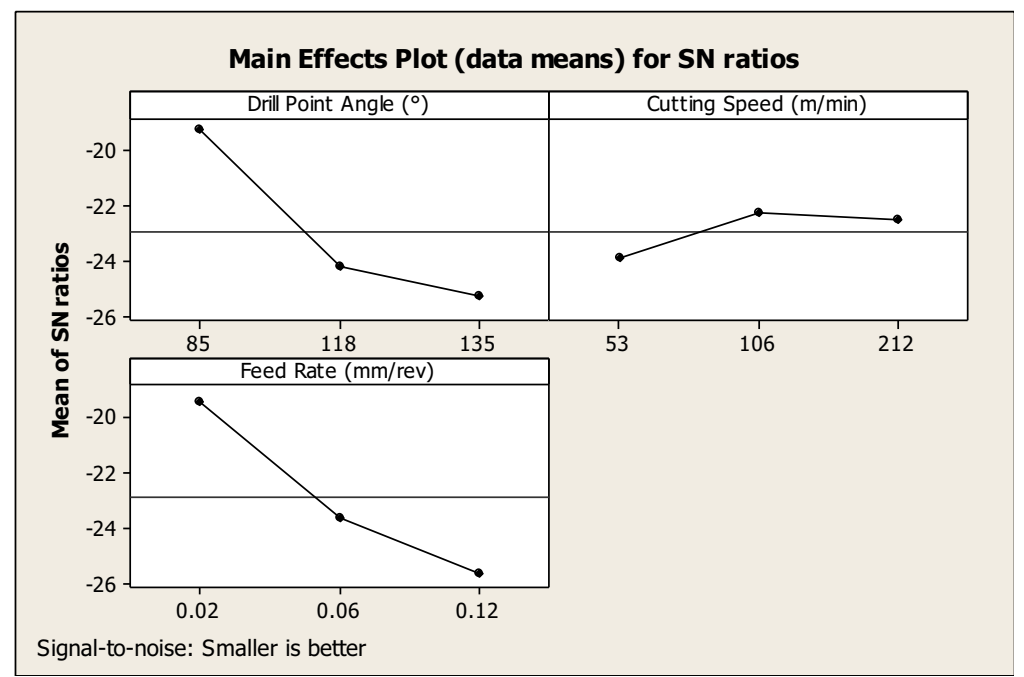

Figure 4. Main effects plote for SN ratios (thrust force)

The reduction of thrust force by using the low feed rate and small drill point angle is observed in contour plot graph (Fig. 5). This graph shows that drilling HFRP composite at $0.02 \mathrm{~mm} / \mathrm{rev}$ feed rate and $85^{\circ}$ drill point angle resulted in lowest range of thrust force $(5-10 \mathrm{~N})$. In fact, the thrust force applied during drilling HFRP composite is assumed not affecting its structure with tensile strength of $419 \mathrm{MPa}$ and flexural strength of $246 \mathrm{MPa}$.

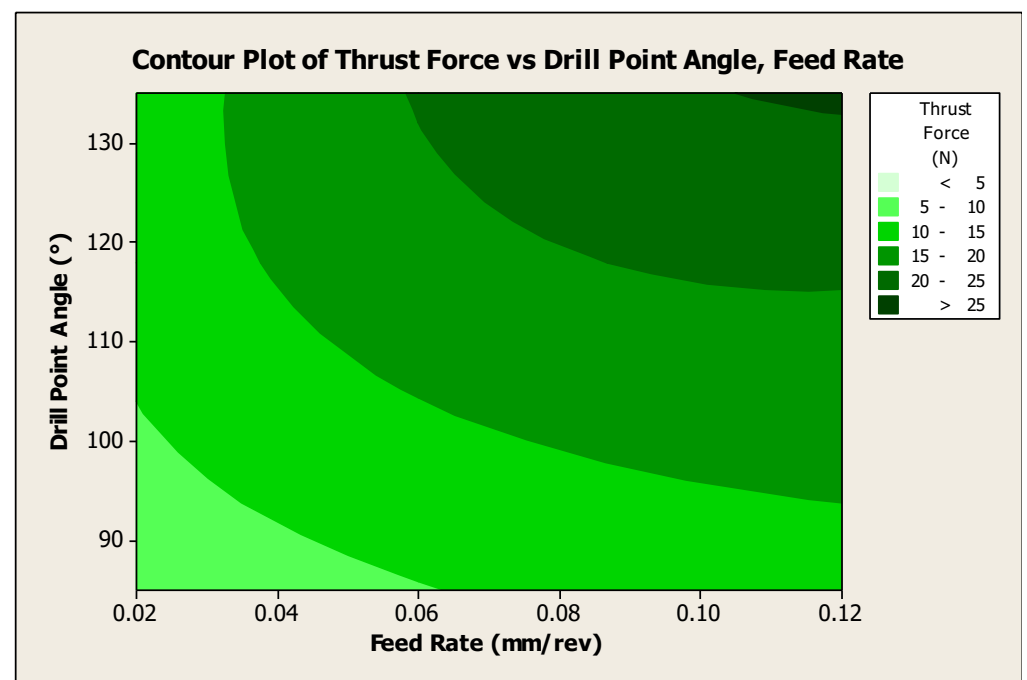

Figure 5 Contour plot of thrust force vs drill point angle, feed rate 
Again, Fig. 5 shows that the range of thrust force gain from this study is almost similar with the range observed by Abrão et al. [2] when he used $118^{\circ}$ point angle of solid carbide drill with 0.04 $\mathrm{mm} / \mathrm{rev}$ feed rate regardless of the material used in his study and yet the similar concept of dynamometer is used to evaluate this response. This shows that the modification done on this dynamometer is acceptable and the optimization of drilling parameters upon thrust force gain from this study is considered valid.

\section{Conclusions}

Full factorial design experiment with the aid of analysis of variance (ANOVA) and signal to noise $(\mathrm{S} / \mathrm{N})$ ratio analysis is able to find the optimum way of drilling HFRP composite through thrust force minimization. In this study, the optimization is achieved when drilling the HFRP composite at low feed rate $(0.02 \mathrm{~mm} / \mathrm{rev})$ with a small drill point angle $\left(85^{\circ}\right)$ and medium cutting speed $(106 \mathrm{~m} / \mathrm{min})$. This optimization process has produced a drilling thrust of $6.4 \mathrm{~N}$ and this value is within $91.89 \%$ confidence upon the confirmation experiment that resulted in $6.92 \mathrm{~N}$. However, the production rate can be increased by using higher cutting speed resulted in higher feed speed as this factor gives a less significant effect as compare to feed rate and drill point angle on the thrust force value.

It is also observed that, this experiment have shown a novel approach by introducing the HFRP composite materials that can have a lower drilling thrust force as compared to glass fiber reinforced polymer (GFRP) composite by only using the conventional drilling tool with small drill point angle at low feed rate and higher cutting speed.

In future, the used of full factorial with the combination of ANOVA approach and $\mathrm{S} / \mathrm{N}$ ratios analysis can be used to find the optimization of drilling parameters by minimizing the delamination factor at the drilled holes entrance and exit. It can be further analyzed the influence of thrust force on the delamination factor of the drilled holes.

\section{Acknowledgements}

Authors would like to thank Universiti Teknologi PETRONAS (UTP) and Production Laboratory, Universiti Teknologi Malaysia (UTM) for the technical and financial supports to this study.

\section{References}

1. C. Dong, and I.J. Davies, Mat. \& Design, 37, 450-457 (2012)

2. $\quad$ A.M. Abrão et al., 29(2), 508-513 (2008)

3. $\quad$ L.M.P. Durão et al.,. 92(7),1545-1550 (2010)

4. U. Heisel and T. Pfeifroth, Procedia CIRP, 1, 471-476 (2012)

5. U.A. Khashaba,. 63(3-4), 313-327 (2004)

6. N.C. Correia et al., Comp. Part A: App. Sci. and Manufact., 36(12), 1645-1656 (2005)

7. C.C. Tsao, Int. J. of Mach. T. and Manufact.,47(7-8), 1132-1138 (2007)

8. C.C. Tsao and H. Hocheng, Int. J. of Mach. T. and Manufact., 45(11),1261-1270 (2005)

9. N.S. Mohan, A. Ramachandra, and S.M. Kulkarni, Comp. Struct.,. 71(3-4) 407-413 (2005)

10. D.C. Montgomery, Design and Analysis of Experiments (New York, John Wiley \& Sons Inc., 2001)

11. W.Y. Fowlkes and C.M. Creveling, Engineering Methods for Robust Product Design Using Taguchi Methods in Technology and Product Development (Canada, Addison-Wesley Publishing Company, 1995) 